\title{
METHODS
}

\section{High-resolution visualization of airspace structures in intact mice via synchrotron phase-contrast X-ray imaging (PCXI)}

\author{
David W. Parsons, ${ }^{1,2,3}$ Kaye Morgan, ${ }^{4}$ Martin Donnelley, ${ }^{1}$ Andreas Fouras, ${ }^{5}$ Jeffrey Crosbie, ${ }^{4,6}$ \\ Ivan Williams, ${ }^{4}$ Richard C. Boucher, ${ }^{7}$ Kentaro Uesugi, ${ }^{8}$ Naoto Yagi ${ }^{8}$ and Karen K. W. Siu ${ }^{4,6}$ \\ ${ }^{1}$ Department of Pulmonary Medicine, Women's and Children's Hospital \\ ${ }^{2}$ Department of Paediatrics, University of Adelaide \\ ${ }^{3}$ Women's \& Children's Child Health Research Institute, Adelaide \\ ${ }^{4}$ School of Physics; ${ }^{5}$ Division of Biological Engineering; and ${ }^{6}$ Monash Centre for Synchrotron Science, Monash University, Victoria, \\ Australia \\ ${ }^{7}$ CF Research and Treatment Center, University of North Carolina at Chapel Hill, NC, USA \\ ${ }^{8}$ SPring-8IJASRI, Hyogo, Japan
}

\begin{abstract}
Anatomical visualization of airspace-containing organs in intact small animals has been limited by the resolution and contrast available from current imaging methods such as $\mathrm{X}$-ray, micro-computed tomography and magnetic resonance imaging. Determining structural relationships and detailed anatomy has therefore relied on suitable fixation, sectioning and histological processing. More complex and informative analyses such as orthogonal views of an organ and three-dimensional structure visualizations have required different animals and image sets, laboriously processed to gather this complementary structural information. Precise three-dimensional anatomical views have always been difficult to achieve in small animals. Here we report the ability of phase-contrast synchrotron $\mathrm{X}$-ray imaging to provide detailed two- and three-dimensional visualization of airspace organ structures in intact animals. Using sub-micrometre square pixel charge-coupled device array detectors, the structure and anatomy of hard and soft tissues, and of airspaces, is readily available using phase-contrast synchrotron X-ray imaging. Moreover, software-controlled volume-reconstructions of tomographic images not only provide unsurpassed image clarity and detail, but also selectable anatomical views that cannot be obtained with established histological techniques. The morphology and structure of nasal and lung airways and the middle ear are illustrated in intact mice, using two- and three-dimensional representations. The utility of phase-contrast synchrotron X-ray imaging for noninvasively localizing objects implanted within airspaces, and the detection of gas bubbles transiting live airways, are other novel features of this visualization methodology. The coupling of phase-contrast synchrotron X-ray imaging technology with software-based reconstruction techniques holds promise for novel and high-resolution non-invasive examination of airspace anatomy in small animal models.
\end{abstract}

Key words airspaces micro CT; airway surface; imaging; mice; non-invasive; phase-contrast; synchrotron; X-ray.

\section{Introduction}

In developing airway gene transfer procedures for the treatment of cystic fibrosis airway disease, we seek new approaches to non-invasive detection of airway health and disease in live mouse models.

\section{Correspondence}

Dr D. W. Parsons, Department of Pulmonary Medicine, Women's and Children's Hospital, 72 King William Road, North Adelaide, South Australia. E: david.parsons@cywhs.sa.gov.au

Accepted for publication 8 April 2008
Although traditional light and electron microscopy histological methods are used to produce high-resolution images and measurements of airway structure in animal models, they require invasive tissue sampling and are usually terminal procedures. In humans, similar levels of histological analysis are possible via invasive biopsy, but often require anaesthesia and are not suited to repeat assessment to track, for example, the durability of a therapeutic treatment. The non-invasive options for intact imaging in humans, such as ultrasound, diagnostic X-ray, high-resolution spiral CT, and standard or hyperpolarizedhelium MRI provide an extraordinary range of complementary 


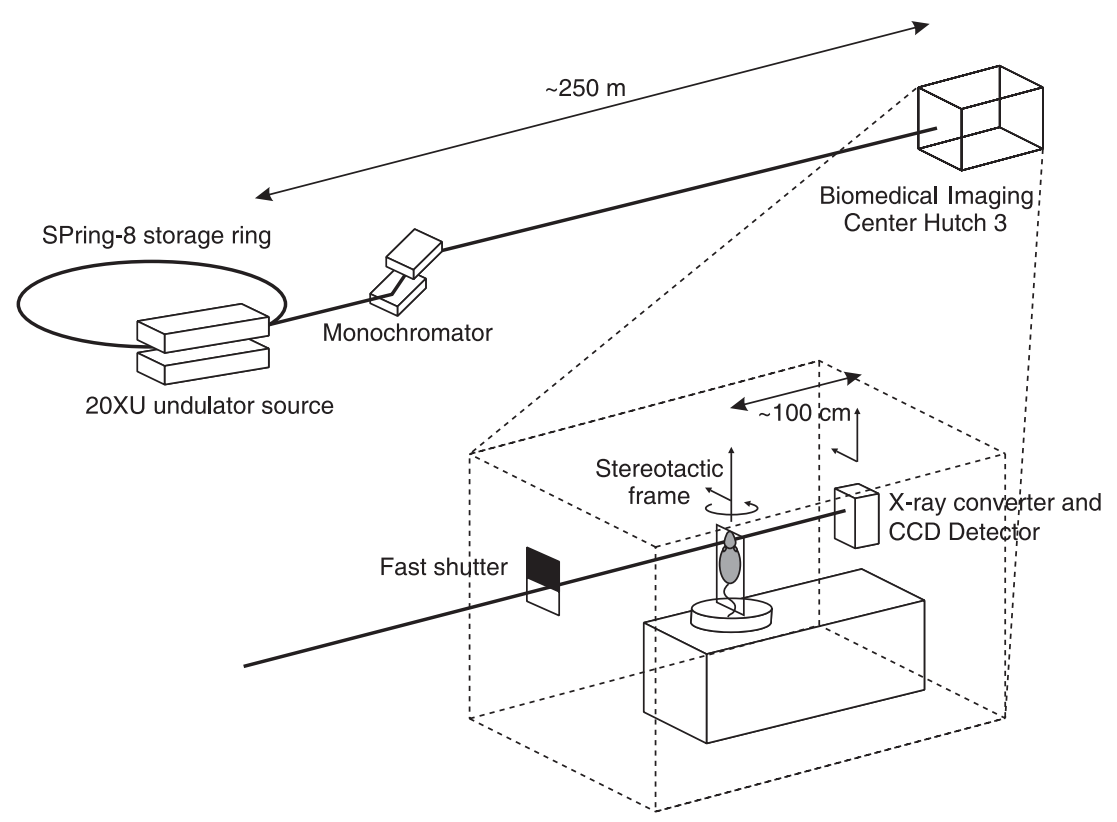

Fig. 1 Arrangement for synchrotron imaging of mouse airway in vivo, or post-mortem (for CT slices). Mice were held vertically and the beam directed through the mouse as required for 2D or CT imaging. Rotation of the stereotactic frame was provided remotely via the baseplate. For CT slice imaging mice were constrained in a plastic tube. information. However, when directed towards small animal model systems, these methods lack adequate contrast and they have poor spatial and temporal resolution. Laboratory X-ray imaging systems designed for small animals provide improved resolution, but airspace structures are poorly detected, contrast remains low, and high resolution scans can involve lengthy image capture and processing times.

Phase-contrast imaging utilizes $\mathrm{X}$-ray refraction effects, in addition to conventional absorption, to create image contrast. It is particularly useful for soft tissue contrast, where the absorption differences are small, and tissue boundaries are enhanced by phase effects due to variations in refractive indices. Propagation-based phase contrast $\mathrm{X}$-ray imaging $(\mathrm{PCXI})$ is the simplest method, rendering phase effects visible as intensity changes by utilizing an increased distance between the sample and the detector (Fig. 1). The technique demands a source with high coherence (i.e. the source size is sufficiently small or, equivalently, the incident $X$-rays may be considered parallel). This requirement is easily realized using a synchrotron $X$ ray source, particularly at long beamlines such as those at the Biomedical Imaging Centre at the SPring-8 synchrotron in Japan (Gotto et al. 2001).

PCXI can provide the substantially increased resolution and contrast needed for non-invasive imaging in small animals. Soft tissues and airspaces such as airways and lungs can now be imaged and processed to give clarity of images that is similar to that provided by bone. Here we reveal the capability of this method for use in anatomical study via two- and three-dimensional imaging in selected mouse airspace organs.

\section{Materials and methods}

Adult C57BI/6 mice weighing 18-20 g were imaged under approvals from the Animal Ethics Committee of SPring-8, and of the Women's and Children's Hospital, CYWHS, Adelaide. All PCXI images were collected at the Biomedical Imaging Centre, SPring-8 Synchrotron facility, Hyogo, Japan. Live mice were anaesthetized with nembutal (45 mg kg-1, i.p.). Post-mortem mice were imaged fresh (Figs 2, 3) or at room temperature after having been kept chilled for up to 2 days $\left(4^{\circ} \mathrm{C}\right.$, and passive rewarming over several hours - Figures and supplementary material numbered 5,6 , and 7 ). Fur produced very strong phase-contrast effects, but removal with depilatory cream prior to imaging improved the clarity of the two-dimensional images.

\section{Two-dimensional airway studies in live and post-mortem mice}

Figure 1 shows the Biomedical Imaging Beamline layout at SPring-8. Post-mortem, or anaesthetized, mice (Fig. 4) were secured headhigh in a stereotaxic frame. The dorsal incisors were hooked over a stainless-steel wire loop and metal bars were positioned at the rear of the skull or in the external ear canal to minimize respiratory movements during live imaging. The torso was supported from below with foam blocks placed under the hindquarters. The depth of anaesthesia was monitored by foot pinch and changes in respiration, and when required, top-up injections were given at half the starting dose of nembutal. Mice remained anaesthetized until humanely killed by nembutal overdose (approximately $500 \mathrm{mg} \mathrm{kg}^{-1}$ i.p.) at the end of each imaging study.

Two dimensional studies were conducted on the undulator Beamline BL20XU where the imaging hutch is located $245 \mathrm{~m}$ from the storage ring. Monochromatic X-rays of $25 \mathrm{keV}(\lambda=0.5 \AA)$ were selected using a standard double-crystal monochromator. At the imaging station, the beam size was approximately $10(\mathrm{H}) \times 6(\mathrm{~V}) \mathrm{mm}$. The restraint frame was mounted on the imaging baseplate, which permitted translations perpendicular to the beam direction 


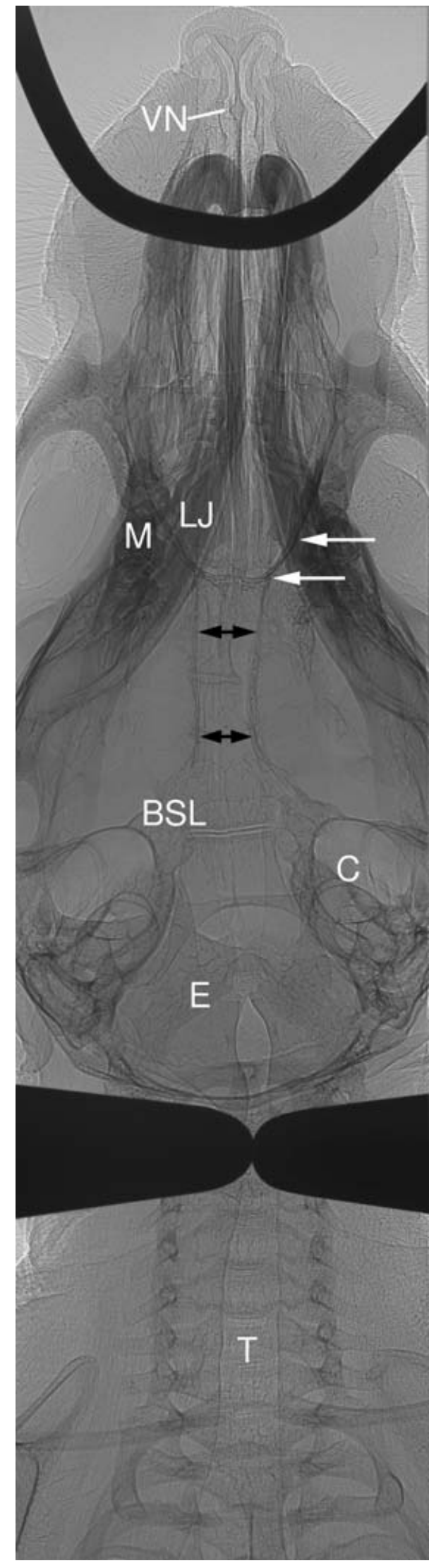

and rotation in the horizontal plane. The beam was directed dorsoventrally through the mouse to image the nasal airways and lower airways, using a propagation (sample to detector) distance of 100$150 \mathrm{~cm}$. Images were captured using a high-resolution X-ray converter (10 mm diameter field of view: AA50 and AA40P Hamamatsu Photonics) with a charge-coupled device (CCD) detector. The converter used a scintillator $\left(\mathrm{Lu}_{2} \mathrm{SiO}_{5}: \mathrm{Ce}\right)$ to convert X-rays to visible light, which was then directed to the CCD using a microscope objective lens. Two detectors were used: C4742-98-24ER $(1344 \times 1024$ with $6.6 \mu \mathrm{m}$ native pixel size, Hamamatsu Photonics), and a pco. 4000 ( $4000 \times 2672$ with $9 \mu \mathrm{m}$ native pixel size, PCO Imaging). By changing a combination of the $\mathrm{X}$-ray converter, its objective lens and the CCD used, effective pixel sizes between $6.6 \mu \mathrm{m}$ (objective lens magnification, $\times 1$, AA40P, C4742-98-24ER) and $0.45 \mu \mathrm{m}(\times 20$, AA50, pco4000) square were available. Exposure times between $100 \mathrm{~ms}$ and $300 \mathrm{~ms}$ were used; with the shorter time it was possible to minimize movement artefact whilst maintaining an adequate signal to noise ratio for the smaller pixel size detector.

In addition, as some regions of interest (ROI) were larger than the CCD field of view the (post-mortem) mice were translated through the beam in a grid pattern to form a raster-scan of multiple images (e.g. Figs 2, 3). All captured images were conventionally corrected for dark-current offset and flat-field non-uniformity before being digitally tiled to form a composite image. A raster scan of $2(\mathrm{H}) \times 6(\mathrm{~V})$ images using the $6.6 \mu \mathrm{m}$ pixel detector allowed viewing of the entire length of the adult mouse head and trachea, whilst similar sized rasters with an effective pixel of $0.45 \mu \mathrm{m}$ (i.e. $\times 20$ magnification, AA50 X-ray converter and pco4000 CCD) were used to view the airways in detail.

\section{Three-dimensional studies - ultra high-resolution CT slices, and volume reconstructions}

Mice were humanely killed using nembutal overdose, then drawn into a securely fitting $(30 \mathrm{~mL}$ ) plastic syringe barrel by a loop of thread secured around their upper incisors. This imaging system limited slow body and organ shifting during the longer imaging periods required for these CT studies (between 30 and $90 \mathrm{~min}$ ). Data were acquired with mice held upright at the bending magnet Beamline 20B2 using a monochromatic beam of $17 \mathrm{keV}$ $(\lambda=0.7 \AA)$ and a propagation distance of $65 \mathrm{~cm}$. In some mice a small diameter polyethylene recording cannula was inserted to a depth of $2.5 \mathrm{~mm}$ or $5 \mathrm{~mm}$ into one nostril, to test the ability to direct the cannula tip to specific regions of airway epithelium. Projection images were acquired using the AA60 X-ray Converter

Fig. 2 Low resolution $(6 \times 6 \mu$ m pixel detector) image of adult mouse post-mortem showing the oral and nasal airways of the head. Nostrils, septum and the ventral septum expansion housing the vomeronasal organ (VN) associated with pheromone detection are clear. The loop of $500 \mu \mathrm{m}$ diameter stainless steel wire suspends the mouse via its upper incisors within the restraint frame. The lower jaw (LJ) and molar teeth (M) are shown. One side of the lateral/posterior edge of the olfactory turbinate region is indicated by white open arrows. Double-ended black arrows indicate the nasopharyngeal airway below the brain. BSL is a ventral bone suture line containing cartilage (see Discussion; a smaller similar suture is present more anteriorly, between the two pairs of black arrows, and is the BSL shown in Fig. 4). Bilateral globular structures are the cochlea (C). In this mouse the trachea (T) is deviated, and its diameter reduced, by the positioning of the two metal fingers used for rear head restraint. $\mathrm{E}$ is the epiglottis at the anterior end of the trachea. 

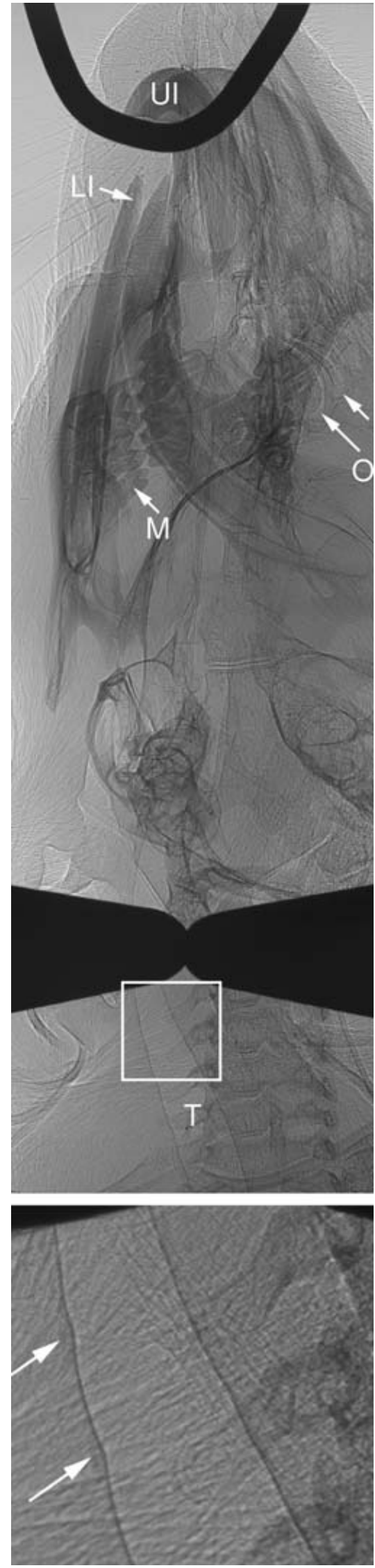

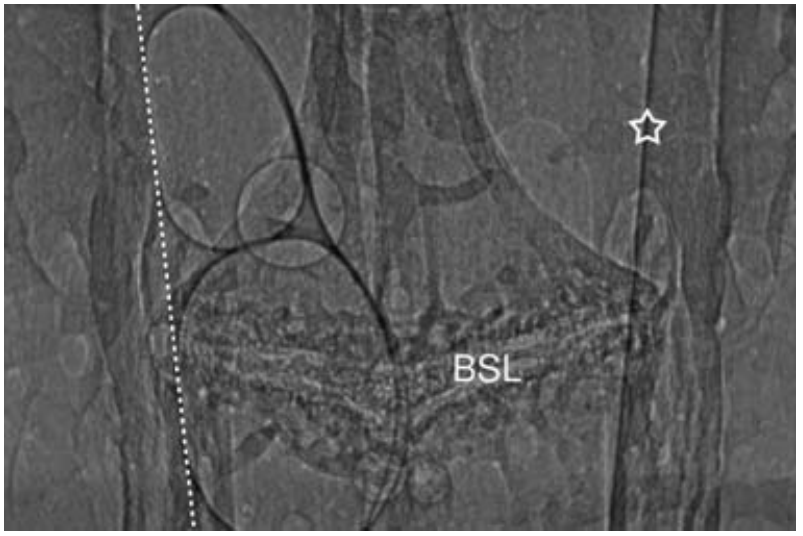

Fig. 4 Live airway: detection of gas bubbles. At 17 min after a $20 \mu \mathrm{L}$ bolus of saline was introduced into the right nostril this image was captured from the nasopharyngeal airway. A distinctive bone suture-line (BSL) with an associated anterior central bilateral curvature provides a landmark for positioning of the imaging beam. Before fluid was instilled, the lumen edge on the left appeared similar to that shown on the right side (star); the original position of the airway surface is shown by the dotted line. At least three bubbles (of many 100s that appeared and transited posteriorly during the live imaging period) are associated on the left wall of the lumen. Two large flattened bubbles are situated adjacent dorso-ventrally to each other, and we propose the one small circular bubble is present separately either in front of or behind the large bubble pair, overlapping the larger bubbles and providing a 'lensing' effect that increases the image intensity inside the bubble area. See Supplementary material Fig. 4.avi for additional information.

( $35 \mathrm{~mm}$ field of view; see http://jp.hamamatsu.com/products/x-ray/ pd450/xrayimag/index_en.html for specifications for this and for the AA50 converter noted earlier) and C4880-41S CCD (Hamamatsu Photonics) in $2 \times 2$ binning mode. This gave a maximum field of view of $2000(\mathrm{H}) \times 1312(\mathrm{~V}) \times 12 \mu \mathrm{m}$ pixels, i.e. $24(\mathrm{H}) \times 15(\mathrm{~V}) \mathrm{mm}$, necessitating $\mathrm{CT}$ acquisition in several vertical sections to collect data for the entire mouse head. CT reconstructions were performed using a Hanning filter and conventional filtered backprojection algorithm without any phase reconstruction techniques, producing a volume with an effective isotropic voxel size of $12 \mu \mathrm{m}$. The intrinsic edge enhancement in the phase contrast projection images means that the reconstructed volumes also demonstrate this characteristic emphasis of boundaries, rendering airway surfaces particularly well without the necessity for further processing. In volume renderings (Software: VolVIEw 2.0, Kitware Inc. USA; and Amira, Mercury Systems) we applied a linear gradient to the greyscale palette for optimal display of these surfaces in the volume rendering.

Fig. 3 Oblique image of the mouse shown in Fig. 2 provides improved image clarity for the trachea ( $T$, arrows) due to elimination of bone from the projection. The lack of interference from the teeth allowed the olfactory turbinates $(\mathrm{O}$, arrows) to be more clearly seen. The presence and structure of the molar teeth (M) and the upper (UI) and lower (LI) incisors are also revealed with greater clarity in this oblique profile. Inset at three-fold magnification shows the tracheal wall, with slight indentations and density increases (two arrows) associated with the tracheal cartilage rings. The presence of these rings is most obvious on the left hand edge of the trachea, and they can also be seen clearly in the rendering shown in Fig. 5B. 


\section{Results}

\section{Two-dimensional imaging - live and post-mortem mice}

Because of our interest in airways, we primarily examined the nose, trachea, and lung. The murine nasal airways are of special interest because of their routine use as a model site for assessing the success of reporter gene and CFTR (cystic fibrosis transmembrane conductance regulator) gene transfer in pre-clinical studies of gene transfer and expression (Grubb \& Boucher, 1999). Nasal airways were well suited to live imaging because they displayed little movement during respiration. In contrast, tracheal imaging could suffer from movement artefact in live animal studies.

Synchrotron PCXI revealed unprecedented detail of the mouse body, and the organs associated with airspaces. Figure 2 (typical of the four mice in that series) shows nasal, tracheal and middle ear airspaces at low resolution in a dorsal-ventral view. The anatomical relationships between the bony structures of the head and the airspaces are readily apparent. The olfactory regions are difficult to see in this view due to the overlap of the jaw, teeth and skull. In these X-ray images, the airway interface was welldelineated in the trachea where surrounding structures did not interfere with the image. Figure 3 is an oblique view of the same mouse, and the trachea is now clearly visible (see inset).

This method is able to resolve individual hairs (see nose tip, Fig. 2) in high contrast. The anatomical relationship of the airspace, the cartilage rings, the soft tissues of the neck, and the bone of the vertebrae demonstrates the power of PCXI to simultaneously visualize a range of tissue types.

\section{Gas bubbles: detection in live airways}

PCXI can detect hitherto unobservable features on and in airways. Bubble presence during normal respiration was produced when a bolus of saline was instilled into the nasal airway of an anaesthetized mouse. A short time later, gas bubbles appeared in the airway, and these continued to transit the field of view for much of the imaging period (Fig. 4, and Fig. 4.avi in Supplementary material). Of interest is that individual and conjoined bubbles, as well as bubble interstices, were all readily apparent. The noninvasive detection of airway surface bubbles within a living animal non-invasively is a unique feature of PCXI that extends the possibilities of the method for high resolution studies and the novel detection of in vivo airway activity.

\section{High resolution CT - two-dimensional and three-dimensional anatomical imaging}

Upper body and head CT studies were performed on 17 mice. The results show that high-resolution $\mathrm{CT}$ imaging via
PCXI produces striking illustrative images of small animals and animal organs. Histology-like thin slices, as well as three-dimensional volume reconstructions with arbitrary viewpoints chosen as desired, are both available.

Figure 5 shows a short series of CT slices through the lung of one mouse, revealing details of bifurcations of airways down to approximately $85 \mu \mathrm{m}$ diameter with slice reconstruction; a representative volume-rendered view of the upper body of another animal is displayed in Fig. 5B. The three-dimensional relationships of the airways, airway cartilage (for trachea), bone, fur, and other anatomical structures are displayed in striking detail and contrast. The image was taken from an animation (see Fig. 5B.avi in Supplementary material for $360^{\circ}$ rotation animation) constructed using VoLVIEW software and clearly shows the spatial relationships between these anatomical structures. To enable volume rendering of this large volume, the dataset size was reduced by $2 \times 2 \times 2$ binning, giving an effective voxel size of $24 \mu \mathrm{m}^{3}$. The azimuth and elevation of the volume can be changed interactively, and quantitative measurements (such as airway width/diameter, and organ size) can be made on screen if desired. Note again the simultaneous presentation of skin, bone, and cartilage, each with clarity and in high detail.

In 10 of the 17 mice we completed CT slice acquisition and volume reconstructions with dummy polyethylene cannulae inserted into the nostril. This was done to simulate the cannula placement used during electrical recording of nasal airway transepithelial potential difference for gene transfer assessment (Fig. 6). Both the CT slices and three-dimensional reconstructions were used to localize the position of the cannula tip after placement at the same insertion depths used in our mouse gene-transfer studies in mice. We found the small diameter $(\sim 300 \mu \mathrm{m})$ heat-thinned cannula tips rested in either the upper or medial nasal airway regions, close to the midline. In contrast to the thinner cannulae, when an unmodified $(610 \mu \mathrm{m}$ diameter) PE10 size cannula was inserted either $5 \mathrm{~mm}$ or $2.5 \mathrm{~mm}$ into the nose, these cannulae (Fig. 6C) were large enough to displace the nasal septum or turbinates physically. When these high resolution CT slices were volume-rendered for one animal (Fig. 6.avi in Supplementary material) the three-dimensional relationship between the cannula and the nostril airways was clearly demonstrated.

\section{Lung airways and gas-exchange regions}

The detailed morphology of the alveolar and conducting airspaces of a mouse lung is shown in the reconstructed CT slices (Fig. 7A). These images provide similar information to that obtainable from human lung $C T$ slice images, though at a much higher resolution $(12 \mu \mathrm{m}$ isotropic voxels compared to the current best possible $\mathrm{CT}$ resolution of approximately $350 \mu \mathrm{m}$ isotropic voxels). Conducting airways, alveolar spaces, and the edges of lung lobes in the 

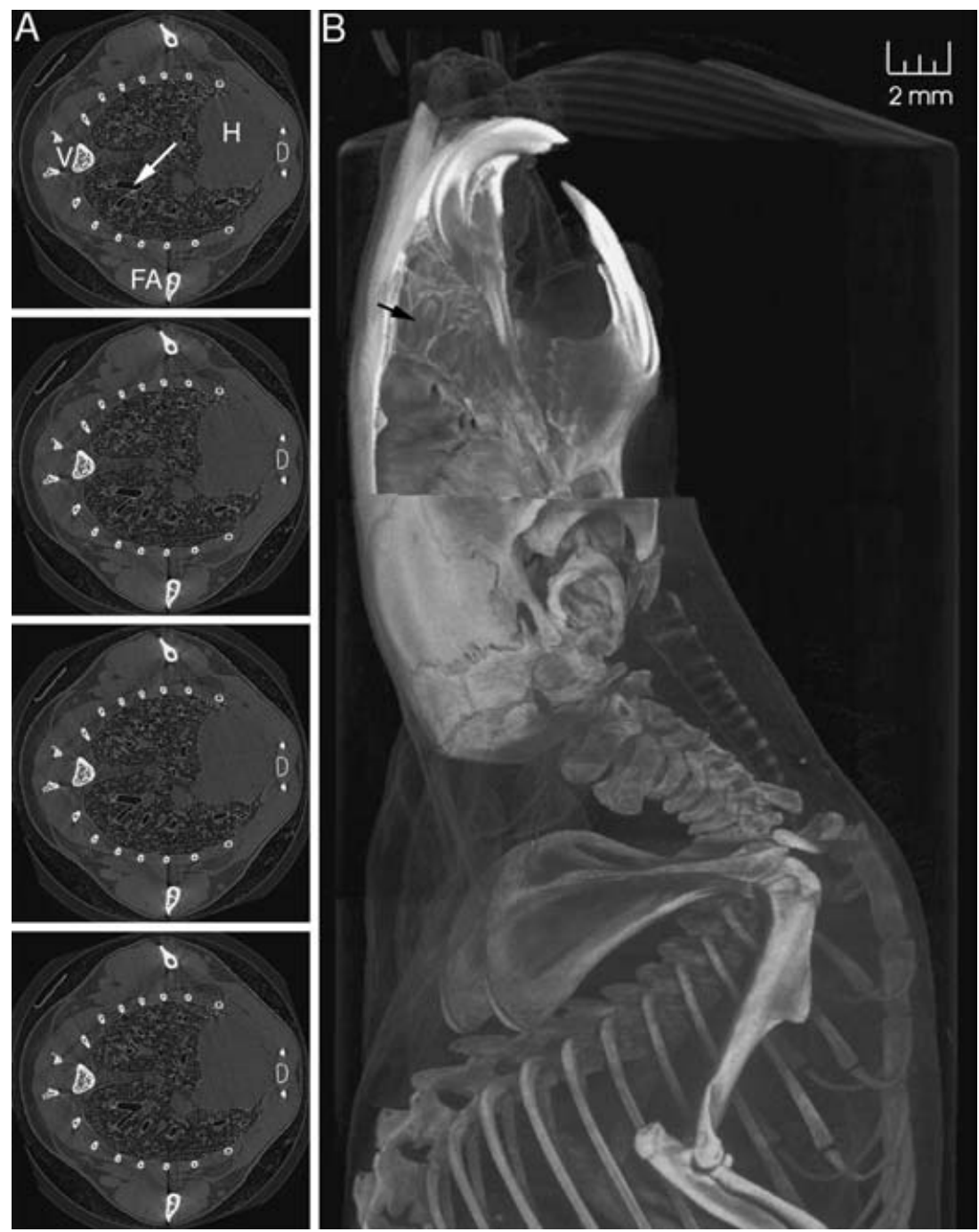

Fig. 5 A) Four consecutive mouse lung $C T$ slices $(2.06 \times 2.06 \mathrm{~cm}$ image with $12-\mu \mathrm{m}$-square pixels and a slice spacing of $12 \mu \mathrm{m}$ ) show a small part of a branching progression (e.g. at arrow) of airways in the lung. The heart $(\mathrm{H})$, forearm bones and musculature (FA), vertebra $(V)$ and the space between the mouse body and the plastic imaging tube are clear. B) Lateral view of the volume-reconstructed mouse ( $12 \mu \mathrm{m}$ isotropic voxels), with software-selected removal of the right upper quarter of the head to reveal detail of the left jaw, molars, and exposed olfactory turbinate region (black arrow). Skull bone sutures lines are also apparent. In the chest region the trachea and associated cartilage rings, sternum, ribcage, spine and shoulder structures are shown. In this instance, the method of restraint in the tube induced hyperextension of the neck. An animation of this dataset is provided in Supplementary material Fig. 5B.avi.

chest cavity can be seen. The muscle bundles that surround the chest are also apparent.

Volume-rendering of CT slices from the lung provided a unique three-dimensional view of selected portions of the lung. The clarity of the airway wall and lumen suggests that airway obstructions produced by mucous accumulation, or disorders of airway wall structure, may be detected non-invasively in rodent models.

The unique visualization abilities of synchrotron PCXI in anatomical studies are further demonstrated in Fig. 7. Starting with $\mathrm{CT}$ slices like those shown in Fig. 5A, different volume-rendered portions of mouse chest, lung, airway, and alveolar regions were produced from the same data. Different representations of the enclosed lung airway and alveolar regions were created using the dataset that produced Fig. 7A (see Fig. 7A-1, 7A-2.avi in Supplementary material). A rendering of the details of lung structure in another orientation provides supporting understandings of the lung structure (Fig. 7B, and Fig. 7B.avi in supplementary material). The relative importance of the types of tissue to be displayed, based on their radiographic intensity, was adjusted using the volume-rendering software.

A CT slice through a mouse middle ear and the corresponding tissue below that slice are shown in Fig. 7C,D. Note that the cross-section in Fig. $7 \mathrm{C}$ was of a single $\mathrm{CT}$ slice, and the uncovering of the three-dimensional organ detail below that slice (in Fig. 7D) was performed by manipulating the volume-rendering software. The mouse remained intact, no dissection or tissue removal was required, and a wide range of other user-selected positions and views can be readily obtained by altering settings in the image-manipulation software. In Supplementary material Fig. 7D.avi extends the appreciation of the normally hidden ear structures via a three-dimensional animation.

The traditional visualization of airspace structure in organisms has relied on destructive cross-sectioning and staining, and this has limited the viewpoints available in each sample. Virtual journeys through these airspaces can now greatly improve the understanding of the organ and its spatial relationships within the body. In Fig. 8.mpg 


\section{Cannula Insertion Depth}
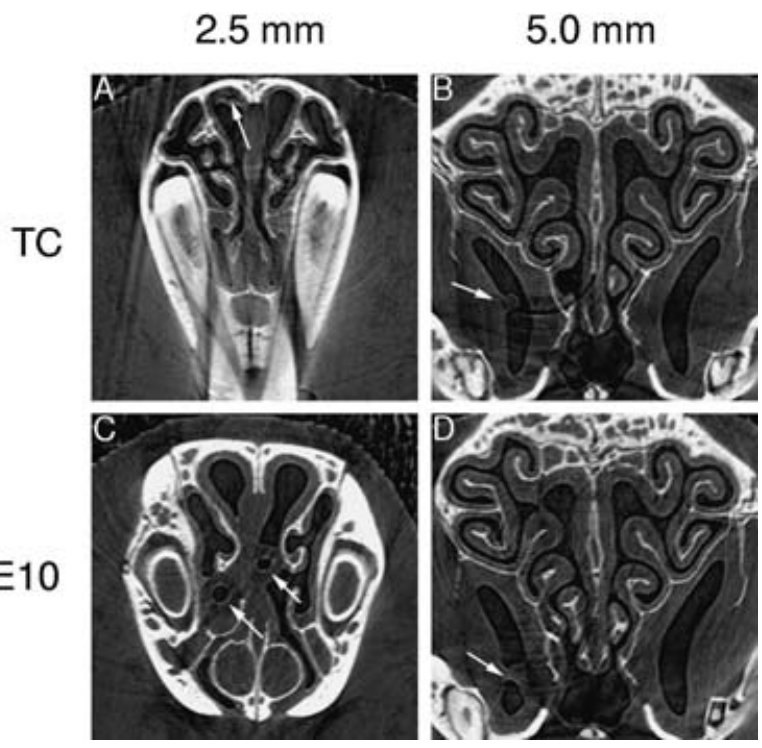

Fig. 6 CT sections of mouse airway showing the position of PE cannulae inserted to specified depths, based on ink markings on the cannula at $2.5 \mathrm{~mm}$ and $5.0 \mathrm{~mm}$ from the tip. Arrows show the cannula location in the nose. For clarity, images are taken approximately four CT slices prior to the tip, but note the cannula tip may appear indistinct (compare Left cannula with Right cannula in C) because of deformation by the cutting blade during tip preparation, and the presence of fluid bridges between the cannula and the airway walls. The ring-like structures in the images are artefacts of the reconstruction algorithm, and derive from a nonuniform response of the detector in certain pixels. Although standard correction procedures are employed to reduce these effects (see Materials and methods) they sometimes cannot correct all artefacts. In five of the 10 animals an inserted cannula (TC) lay in the upper nasal airway (e.g. in A), the remaining five lay in the medial airway adjacent to the septum (similar to that shown for the left-side PE10 size cannula in C). Both the thinned (B) and PE10 cannula (D) could enter the boomerang-shaped maxillary sinus when inserted to the nominal $5 \mathrm{~mm}$ depth; in D the cannula is filled with fluid and neither the lumen nor the cannula walls can be seen within the fluid bridge produced. TC, heatpulled thinned cannula; PE10, standard diameter PE10 polyethylene cannula. A volume-rendered animation of a thinned-cannula placement in the mouse nose is provided in supplementary material (Supplementary material Fig. 6.avi file).

(Supplementary material) an example 'virtual journey' through the mouse nose and lung has been constructed using suitable software (Amira; see Materials and methods) and is able to provide user-definable virtual travel into and through the airspaces.

\section{Discussion}

The inherent high coherence and intensity of synchrotron X-ray sources allows the exploitation of phase-contrast effects to enhance airway edge visibility well above that possible from conventional $X$-ray imaging and can do so at a potentially lower dose of radiation (Lewis, 2004), at least for two-dimensional imaging. The intrinsic high brightness of X-rays from a synchrotron source - typically many orders of magnitude greater than conventional sources - coupled with fast, high resolution detectors, provides a method of imaging that has shown considerable potential for high resolution imaging of airways and lungs. Furthermore, no contrast agents or other invasive procedures are required.

With the improvements in digital image-capture technology and storage, and technical advances in the control and use of synchrotron X-rays for biological and medical imaging, PCXI imaging studies of animals and organs have begun to appear. The first demonstration of the phasecontrast methodology by Snigirev (Snigirev et al. 1995) and Wilkins, and the subsequent use by other groups (Wilkins et al. 1996; Yagi et al. 1999; Suzuki et al. 2002; Westneat et al. 2003; Sera et al. 2005) has demonstrated its technical simplicity, with only a separation between sample and the detector needed to provide substantial contrast improvement. The ability to detect tissue types that could not be easily revealed with conventional X-rays is reviewed by Lewis (2004). Of some interest to respiratory biologists is the ability to detect airway cartilage (see Fig. 5B), a tissue that is largely invisible using conventional $\mathrm{X}$-rays. Figure 2 demonstrates cartilage well, as it reveals a ventral skull suture that can otherwise only be detected histologically. This suture separates the basi-sphenoid and basi-occipital bones; it is formed from the somatic mesoderm, which produces a sandwich of cartilage in the suture line. In contrast, the flat bones of the skull are formed from neural-crest derived neuroectoderm, which produces purely bony sutures that are separated by the types of sutures easily seen on the skull surface (Fig. 5B).

One of the early significant applications of PCXI to the biological anatomy and physiology of small airspaces was that reported by the Westneat group (Westneat et al. 2003; Socha et al. 2007). Their study superbly revealed the detailed anatomy of the tracheal system of a $1.5-\mathrm{cm}$ carabid beetle, in situ, and their in vivo studies provided the first evidence of tracheal pumping in this species. In small insects and organisms less than $1 \mathrm{~mm}^{3}$, a recent European study has comprehensively described the basis and specific techniques for PCXI imaging of muscle groups and tissues in preserved samples (Betz et al. 2007).

Since the studies of lung airway using synchrotron phase contrast have appeared (Yagi et al. 1999; Suzuki et al. 2002; Sera et al. 2003), these techniques have been adopted by others interested in lung anatomy and physiology. More recently, the short exposure times and the high contrast between airspace and tissue have been used to capture extraordinary images in rabbit pups, showing the first in-vivo demonstration of lung aeration and lung liquid clearance at birth (Hooper et al. 2007).

The combination of imaging with histological techniques has similarly progressed, and this combination offers unique opportunities for anatomical studies. Co-registration of 

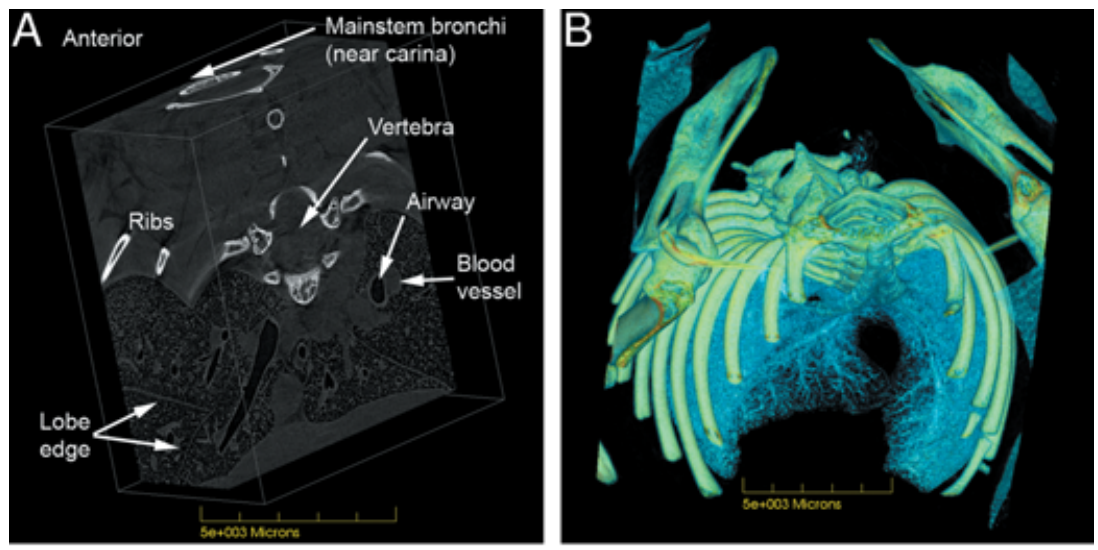

Fig. 7 Chest and lung images; middle ear. A) Volume reconstruction of a block of lung tissue, derived from cropped CT slices. In this representation, bone, airway, blood (including heart), interstitial tissues and gas exchange regions are all visible. See Supplementary material Fig. 7A.avi and Fig. 7B.avi for the associated animations using different software settings able to reveal other anatomical features and relationships that cannot be represented using static images. B) Mouse chest cavity, rendered to optimize visualization of bone and lung airways. The associated animation in Supplementary material (Fig. 7B.avi)
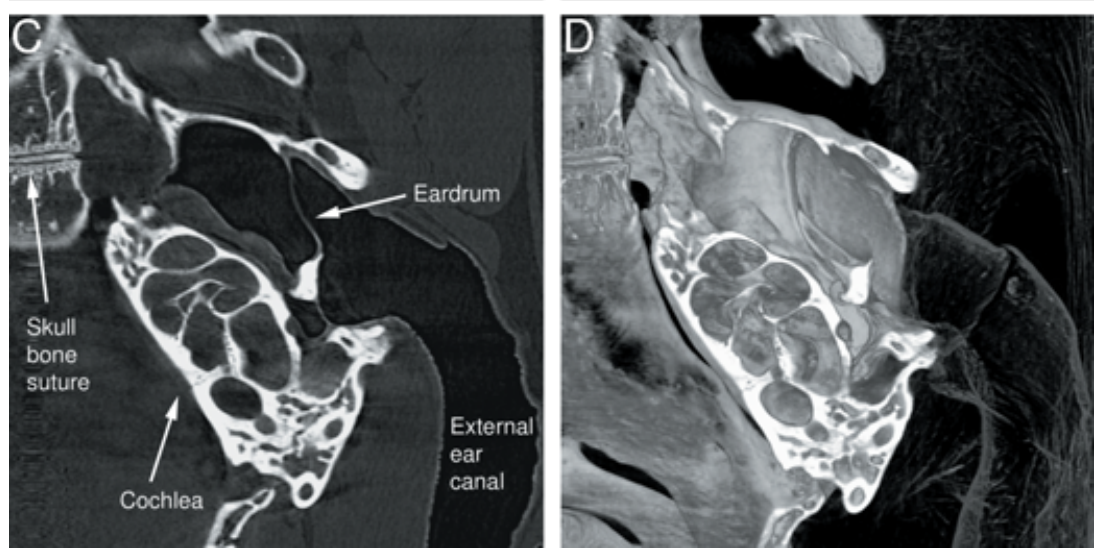
dynamically illustrates the organization of the airway branching pattern amongst the lightly rendered lung lobes and alveolar tissues. C) The middle ear spaces and the cochlea are shown in one 12- $\mu \mathrm{m}$-thick CT slice. D) Volume reconstruction of the middle-ear region on one side of the section shown in Fig. 7C enhances appreciation of the anatomy of the organ and the external ear canal. In Supplementary material (Fig. 7D.avi) a $360^{\circ}$ animation of this reconstructed region provides an even higher level of understanding of the complexity and interrelatedness of the structures of the external and middle ear. The width of the imaged area is $7.2 \mathrm{~mm}$.

sample coordinates across X-ray and histological methods has produced the 'Digimouse' atlas (Dogdas et al. 2007). Although conventional X-ray images were used in the Dogdas study, resulting in far lower resolution and contrast than is available with synchrotron PCXI, the resulting system for identification and viewing of the intact spatial relationships of organs and tissues provides a novel tool that could be applied to visualization and understanding of airspace organs.

Small-animal laboratory micro-CT systems are approaching the resolution levels suitable for preclinical study in small animals like mice, and might be considered for imaging similar to that described here. However, laboratory $\mathrm{X}$-ray radiation is polychromatic and cannot provide the direct quantification that is possible with monochromatic synchrotron X-ray sources. Furthermore, polychromatic light gives lower effective resolution when used in conjunction with phase contrast imaging.

\section{Resolution of airspace edges - bubble wall detection}

Based on the Nyquist sampling theorem, in these studies the maximum resolution achievable using a $0.45-\mu \mathrm{m}$-square pixel detector array (Fig. 4) in these studies is theoretically $\sim 1 \mu \mathrm{m}$. However, scattering in the phosphor scintillator and (objective lens) optics that convert the X-rays to visible light for recording by the CCD produce a practical limit of $\sim 2 \mu \mathrm{m}$ here. Accordingly, at least four adjacent pixels are needed reliably to detect the smallest difference in contrast created by a real anatomical structure.

Figure 4 shows the ability of PCXI to resolve gas bubbles in situ. Based on interferometry analyses of soap-bubble films in visible light, a bubble wall thickness is likely to be less than several $100 \mathrm{~nm}$, and thus is well below the direct resolution of the detector system here. However, PCXI is able to detect bubble walls due to the phase contrast effects that the latter produce. Effectively, PCXI 'inflates' the width of the bubble wall because the wall offers extreme differences in refractive index and thus phase contrast: between the air inside the bubble, across the nanometre-thin fluid of the bubble wall, and then to the airspace outside the bubble wall. The resulting image is a wider representation of the real bubble wall with high contrast that is consequently highly visible. These airway bubble images were captured in live anaesthetized mice, and appear to be the first report of non-invasive detection of airway-surface bubbles in vivo.

By comparison with the unaffected right side of the airway, the gas bubbles detected on the live airway surface (Fig. 4, and see also Supplementary material Fig. 4.avi) show novel features of surface-bound bubble behaviour in live airway. The flattened bases of the two larger-diameter bubbles indicate their dynamic distensibility when embedded within a smoothly enveloping fluid mass on the airway surface. The darkening of image intensity in the interstitial 
fluid-filled space between the large bubbles (where the small circular bubble overlaps them, see Fig. 4) indicated the excellent sensitivity of PCXI to the additional fluid present there.

\section{Imaging airspace surfaces}

The ability to reveal airway surfaces and bubbles lodged along the airway surface is unique to PCXI. The strong contrast between the air, fluid and tissue layers permits non-invasive detection of airway diameter, and thus airway narrowing or obstruction could be detected in animal-disease models. These two-dimensional and three-dimensional techniques provide complementary information about airspaces in these small animals. Our two-dimensional studies in live airways have been able to reveal the dynamic activity in major airways, such as bubbles transiting airways after fluid instillations.

Three-dimensional CT studies in post-mortem animals can reveal striking anatomical detail that is very difficult to achieve in any other way. In deep lung airways the resolution was sufficient to display small conducting airways to approximately $80 \mu \mathrm{m}$ in diameter. With such resolution, changes in diameter due to a disease-based mucus accumulation, physical object obstruction, or an inherited disorder, could be easily identified. The clarity of the mouse cochlea (Fig. 7C,D) exceeded that reported recently using the diffraction enhanced imaging (DEI) method of phase contrast in much larger preserved guinea-pig cochlea specimens (Gao et al. 2006). There are several reasons for the improved visualization here. The detector uses pixels of 10 -fold better resolution; the X-rays are detected under phase-contrast conditions that boost edge detection and detail; and the cochlea remains in situ, thus preserving spatial relationships within the mouse ear. Furthermore, DEI is reliant on using a perfect crystal analyser to render the phase differences visible as intensity differences. Although DEI is a more sensitive method than propagation-based phase contrast in theory, in practice the crystal is prone to stability problems and has a limited field of view compared to propagation-based methods.

Our results show that three-dimensional PCXI imaging can reveal considerable detail about the spatial relationships of organ structures, and should provide useful supplementary information to support and sometimes surpass the capabilities of traditional dissection and histological cross-sectioning to obtain structural information from small animals.

\section{An application of PCXI: localization of recording electrode position in airways}

Physiological recordings of airway electrical potential difference from airways and airspaces in small animals are complicated by the inability to know exactly where a recording electrode is placed. When recording airway potential difference, researchers can only rely on cannula placement according to the depth of insertion into the nose. It is not possible to know where the recording tip rests within this complex structure, and thus whether recorded signals come from the desired regions of ciliated airway epithelium. In a previous study we noted that correct depth placement of the cannula tip in the nasal airway was important (Parsons et al. 2000). These new images, however, provide evidence of the variability inherent in this recording method. Because cannula tips were localized to the respiratory epithelium of either the upper (Fig. 6A) or the medial (Fig. 6C) nasal airway equally often in our studies, and as the deeper upper regions of epithelium are exclusively olfactory, this finding confirms our earlier data, using electrochemical marking of tip position (Parsons et al. 2000), that a shallow $(2.5 \mathrm{~mm}$ ) recording depth is more likely, but not certain, to access nasal respiratory epithelium. We also noted cannulae inserted to $5 \mathrm{~mm}$ could enter the maxillary sinus (Fig. 6B,D) where the entire surface is known to be ciliated respiratory epithelium. These PCXI studies have thus identified the possibility for cannulations of mouse sinuses. Given the complex topography of the mouse nose this possibility could not have been predicted before, nor readily tested using invasive dissection techniques. If this type of sinus access is confirmed in further studies, the maxillary sinus may have utility for the creation, study and manipulation of sinus disorders and disease in mouse models.

\section{New options for visualizing animal airspaces}

The imaging potential offered by the combination of PCXI and current image manipulation software is apparent in Figs 7 and 8. The images in Fig. 7 were taken from one animal, with the different organs, views, colouring, detail, and ability to return to the dataset for new visualizations all controlled by the user.

Supplemental material Fig. 8.mpg suggests one of the novel directions now available for representation of anatomical and morphological analyses in small animals, echoing the developing virtual bronchoscopy techniques within human CT analyses (Finkelstein et al. 2004; Bauer \& Steiner, 2007). Exploration of normally inaccessible or hidden airspaces such as sinuses, nasal airways, and the airways of the lung is possible at resolutions already sufficiently high to detect obstructions and congenital defects noninvasively in intact small animals such as mice. With appropriate beamline and detector technology in place, larger organisms and animals could also be studied in this way.

\section{Concluding remarks}

Our findings show that synchrotron PCXI can provide several complementary visualization approaches able to 
improve anatomical, biological, and physiological studies in small animals. High resolution two-dimensional images of airspace structures in living or deceased animals (such as those shown in Figs 2-4) can be readily produced. Animations and 'virtual tours' based on the three-dimensional reconstructions (Fig. 8) strengthen the understanding of the spatial relationships within and around the organs studied. Given the high resolution and clarity achievable now, the expected improvements in detectors, data capture and image processing, advances in these techniques for use in small animal models and live non-invasive imaging of airspace biology can be expected.

The unprecedented structural contrast and resolution available from PCXI CT slicing offers researchers near histological-quality sections and resolutions of complex organs such as lung (Figs 4, 5A). With volume-reconstructions the overall structure of organs can be interactively viewed intact or cut away from any angle (Figs 5B, 6B, 7A, B). Tailoring the parameters of the reconstruction software allows specified regions of interest to be rendered with emphasis on different components of the tissue (e.g. conducting airway vs. gas-exchange regions) if tissue absorption or phase-contrast properties permit. Although these studies were developed in mice we speculate that with technical advances and tailoring of radiation dose, PCXI may eventually have a role in specialized diagnostic imaging studies at very high resolution in larger animals, and potentially in humans. With a recent report providing the basis for development of a compact laser-based synchrotron X-ray source (Schlenvoigt et al. 2008) the utility of PCXI in biology and medicine for imaging investigations at the tissue, organ and organism level may become substantial.

\section{Acknowledgements}

Studies supported by NH\&MRC Australia, USA CF Foundation, \& philanthropic donors. SPring-8 experiments completed under proposals J05A20XU-0533N, 2006A1066, \& 2007A1287. Prof. Ian Gibbins, Flinders University, advised on the identity and embryology of the cartilaginous skull-bone sutures. Dr Peter Self, Adelaide Microscopy, provided advice and datasets concerning non-synchroton X-ray imaging. D.W.P., K.K.W.S., J.C., and I.W. acknowledge the support of the Access to Major Research Facilities Program, Commonwealth of Australia.

\section{References}

Bauer TL, Steiner KV (2007) Virtual bronchoscopy: clinical applications and limitations. Surg Oncol Clin N Am 16, 323-328.

Betz O, Wegst U, Weide D, et al. (2007) Imaging applications of synchrotron X-ray phase-contrast microtomography in biological morphology and biomaterials science. I. General aspects of the technique and its advantages in the analysis of millimetre-sized arthropod structure. J Microsc 227, 51-71.

Dogdas B, Stout D, Chatziioannou AF, Leahy RM (2007) Digimouse: a 3D whole body mouse atlas from CT and cryosection data. Phys Med Biol 52, 577-587.

Finkelstein SE, Summers RM, Nguyen DM, Schrump DS (2004)
Virtual bronchoscopy for evaluation of airway disease. Thorac Surg Clin 14, 79-86.

Gao X, Luo S, Yin H, et al. (2006) A micro-tomography method based on X-ray diffraction enhanced imaging for the visualization of micro-organs and soft tissues. Comput Med Imaging Graph 30, 339-347.

Gotto K, Takeshita K, Suzuki Y, et al. (2001) Construction and commissioning of a 215-m-long beamline at SPring-8. In 7th International Conference on Synchrotron Radiation Instrumentation, Nuclear Instruments \& Methods in Physics Research, Section A (Accelerators, Spectrometers, Detectors and Associated Equipment), pp. 682-685.

Grubb BR, Boucher RC (1999) Pathophysiology of gene-targeted mouse models for cystic fibrosis. Physiol Rev 79, S193-S214.

Hooper SB, Kitchen MJ, Wallace MJ, et al. (2007) Imaging lung aeration and lung liquid clearance at birth. FASEB J 21, 3329-3337.

Lewis RA (2004) Medical phase contrast x-ray imaging: current status and future prospects. Phys Med Biol 49, 3573-3583.

Parsons DW, Hopkins PJ, Bourne AJ, Boucher RC, Martin AJ (2000) Airway gene transfer in mouse nasal-airways: Importance of identification of epithelial type for assessment of gene transfer. Gene Therapy 7, 1810-1815.

Schlenvoigt H-P, Haupt K, Debus A, et al. (2008) A compact synchrotron radiation source driven by a laser-plasma wakefield accelerator. Nature Physics 4, 103-133.

Sera T, Fujioka H, Yokota H, et al. (2003) Three-dimensional visualization and morphometry of small airways from microfocal X-ray computed tomography. J Biomech 36, 1587-1594.

Sera T, Uesugi K, Yagi N (2005) Refraction-enhanced tomography of mouse and rabbit lungs. Med Phys 32, 2787-2792.

Snigirev A, Snigreva I, Kohn V, Kuznetsov S, Schelokov I (1995) On the possibilities of X-ray phase contrast microimaging by coherent high-energy synchrotron radiation. Rev Sci Instrum 66, 5486-5492.

Socha JJ, Westneat MW, Harrison JF, Waters JS, Lee WK (2007) Real-time phase-contrast $x$-ray imaging: a new technique for the study of animal form and function. BMC Biol March, 6-21.

Suzuki Y, Yagi N, Uesugi K (2002) X-ray refraction-enhanced imaging and a method for phase retrieval for a simple object. $J$ Synchrotron Radiat 9, 160-165.

Westneat MW, Betz O, Blob RW, Fezzaa K, Cooper J, Lee W (2003) Tracheal respiration in insects visualized with synchrotron X-ray imaging. Science 299, 558-560.

Wilkins SW, Gureyev TE, Gao D, Pogany A, Stevenson AW (1996) Phase-contrast imaging using polychromatic hard X-rays. Nature 384, 335-338.

Yagi N, Suzuki Y, Umetani K, Kohmura Y, Yamasaki K (1999) Refraction-enhanced $\mathrm{x}$-ray imaging of mouse lung using synchrotron radiation source. Med Phys 26, 2190-2193.

\section{Supplementary material}

The following supplemental material is available for this article:

Fig. 4.avi Example of gas bubbles detected non-invasively whilst transiting intact live airway. The first six frames (all frames were taken $10 \mathrm{~s}$ apart) show $1 \mathrm{~min}$ of baseline images prior to fluid instillation. After four separating black frames, the 19 following frames reveal a range of bubble sizes and groups moving down the airway from the 
overlying nostril region towards the pharyngeal area, over a 3-min period. Individual and linked bubbles as well as the rapidly changing fluid level associated with the bubbles is apparent. Frame 18 and the scale are the same as Fig. 4.

Fig. 5. avi Animated view of rendered head and chest. Views of the skull, neck and chest from all horizontal directions show with greater clarity the features noted in Fig. 5B. This particular digital sectioning and rotation permits viewers to simultaneously appreciate the detail of skull-bone suture lines, the detail within the cutaway section exposing the olfactory turbinate region, and the soft tissues of the outer ears and neck.

Fig. 6.avi Non-invasive localization of cannula in upper nasal airway. The cannula is visible entering the nasal orifice (in frame 1 the cannula has been overlaid in red), and can be tracked though the nostril to its termination in the nasal airway. The white nasal-bone can be seen dorsally, where it terminates before reaching the nose tip. Similarly, the (white) anterior portion of the dorsal incisor is present ventrally. Note also the resolution of hair of the fur on the nose outer surface.

Fig. 7A-1.avi This animation was produced from the same dataset that provided the image of Fig. 7A, but here is shown with a different orientation and with progressive magnification. It was rendered to be similar to the view of a block of fixed lung tissue produced by simple right-angled cuts with a blade. At the top of the first frame the base of the trachea and the carina with the two mainstem bronchi are shown; note the regular bilateral whitened regions of airway cartilage on the airway wall. With rotation past the side of the lung some medium-size airways, and the edges of two lung lobes can be seen (see Fig. 7A for labeling). With further rotation the left and right sides of the lung, the continuation of the lobe edge, and the large airways that connect back to the carina become apparent. The last frame shows three smaller airways, mostly in cross-section, with their accompanying blood vessels.

Fig. 7A-2.avi This animation is the same dataset, orientation, rotation and magnification as in animation 7A-1.avi, but with rendering and coloration designed to separate out airways and bone. The dorso-posterior sweep of the mainstem bronchi is apparent as they connect into the lung lobes, and the digital removal of blood from the lung can be seen in the empty blood vessel adjacent to the large airway right of centre in the last frame. This rendering has also removed the heart and associated thoracic tissues, leaving the lung tissue largely intact. Note that some lung lobe edges are fragmented due to the effect of digital region cropping when digitally producing this block of chest tissue for viewing.
Fig. 7B.avi An anterior to posterior rotation animation, again using the same dataset and with rendering settings assigned as for 7A-2.avi, but starting from an anterior viewpoint. However, the degree of cropping has been relaxed, to include more of the ribs and the full posterior extent of the lungs. At Frame 50 a small structure below the lungs appears, and was found to be in the position of the stomach in other renderings (data not shown). The structure here represents the top of the stomach, containing an air bubble and food material (follow to the last frame for more clarity). By Frame 130 the medio-ventral edge of a right lobe is silhouetted against the background.

Fig. 7D.avi Slow $360^{\circ}$ rotation ( $2^{\circ}$ increments) of the ear dataset rendered in image Fig. 7D. The relationships amongst the outer ear, ear canal, eardrum region, middle-ear bony structures and the cochlea are seen in fine detail. Scale as in Fig. 7D.

Fig. 8.mpg Virtual tour of mouse nose and lung reconstructed from CT slices. This is the same mouse as that used for Fig. 6C. Beginning with a view of the posterior edge of the $C T$ slice series, sections through the ribs, forearms, and the anterior end of the stomach (containing food material) can be seen. Rotation to the nose tip displays the mounting tube, the animal with its outer ears laid flat, and the skull, teeth, and whiskers. The two polyethylene cannulae (see Fig. 6C) can be seen in the nostrils, and once the view has progressed inside the nose, two views of the upper nasal turbinates are seen (frames 380-480). The viewpoint progresses posteriorly along the left nasal airway until (frame 670 ) the scene scans to ventral airspaces before crossing to the right airway region at this same depth. From the right nasal airway (frame 960) the view moves posteriorly with the septum on the right, until centralizing in the nasopharynx (frame 1200). The larynx and trachea come into view (frame 1220) with the viewer taken down into the trachea, past the carina (frame 1510), and into the right bronchus and deeper airway branches. At frame 1820 the view passes through the airway wall, exits the base of the lung, and rotates to reveal the stomach, ribs and forearms of the first frame (permitting continuous loop animation).

This material is available as part of the online article from: http://www.blackwell-synergy.com/doi/abs/10.1111/ j.1469-7580.2008.00950.x

(This link will take you to the article abstract).

Please note: Blackwell Publishing is not responsible for the content or functionality of any supplementary materials supplied by the authors. Any queries (other than missing material) should be directed to the corresponding author for the article. 